\title{
An Approach for Externalization of Expert Tacit Knowledge Using a Query Management System in an E-Learning Environment
}
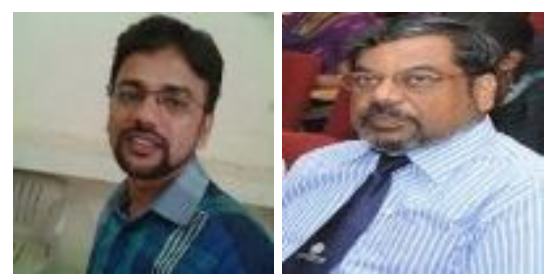

Abdul Azeez Khan and Sheik Abdul Khader B.S.Abdur Rahman University, India

\section{Abstract}

E-learning or electronic learning platforms facilitate delivery of the knowledge spectrum to the learning community through information and communication technologies. The transfer of knowledge takes place from experts to learners, and externalization of the knowledge transfer is significant. In the e-learning environment, the learners seek subject expertise to clarify their subject queries, and a learner query can be routed to an expert for externalization of expert knowledge provided the learner knows the subject expert or the expertise group. However, learners new to e-learning systems are not aware of the expertise group to which the query should be sent, which results in time delays, non-response, inaccurate solutions and loss of knowledge capture. Several models have been proposed to resolve this task, but thus far, these efforts have focused completely on returning the most conversant people as experts on a particular topic to retrieve valuable knowledge. To address this problem, we propose an approach that externalizes the tacit knowledge of a subject expert by creating a dynamic query handling system that automatically transfers a user query to the best subject expert.

Keywords: E-learning system; expert finding; knowledge management; tacit knowledge; knowledge sharing; knowledge capture 


\section{Introduction}

E-learning, or internet-enabled learning, uses internet technologies to deliver a broad array of solutions that enhance knowledge and performance. The Internet has begun to reshape education approaches with many versions of e-learning software used extensively at various levels of education, that is, universities, high schools, vocational schools, or junior levels. E-learning provides multiple benefits beyond conventional classroom-based learning (Manongga, Utomo, \& Hendry, 2014).

The traditional context of learning is currently undergoing a drastic change. Many situations exist in which learners desire to study specific topics in which they are interested without the constraints of time and place. These needs require that learning be personalized, flexible, and available on-demand. In the corporate sector, a heavy focus is turned towards sharing of knowledge between experienced and inexperienced users or new employees. Institutions also focus to a greater extent on sharing of knowledge between experts and learners.

Advances in computer technology in the recent decades have significantly transformed modern teaching approaches. Systems providers have developed different types of elearning tools that ease the learning process and improve outcomes. Higher education institutions are using the benefits of these e-learning tools to design and offer new opportunities for teaching and learning. To evaluate the success of one type of $\mathrm{e}$ learning tool relative to another, we must understand whether a specific type of elearning tool can effectively support the learning process. Therefore, it is important to determine whether the tools can provide feedback and continuously refine the learning process; contain individual characteristics, needs, learning styles, and learning pace; and deliver high-quality information through a suitable medium to create a sense of personal touch and support. E-learning tools can be considered as a digital medium that facilitates information transfer between knowledge sources (instructors) and knowledge seekers (students). To evaluate the proposed work, we examined two leading e-learning tools, namely, Blackboard and Moodle, in a comparison of communication aspects provided for the knowledge seeker and knowledge provider. 
Table 1

Comparison between Blackboard and Moodle

\begin{tabular}{|l|l|l|}
\hline $\begin{array}{l}\text { Communication } \\
\text { Tools }\end{array}$ & Blackboard & Moodle \\
\hline Chat & $\begin{array}{l}\text { Tool with an instant messaging } \\
\text { option will send messages, provided } \\
\text { the recipient is available online. }\end{array}$ & $\begin{array}{l}\text { Instant messaging and } \\
\text { chat option with online } \\
\text { users. Offline messages } \\
\text { enabled. }\end{array}$ \\
\hline Email & $\begin{array}{l}\text { Email can be sent to all students or } \\
\text { to those in the subset workgroup. }\end{array}$ & $\begin{array}{l}\text { Instant messaging tool can } \\
\text { send messages to offline } \\
\text { users, and messages can be } \\
\text { read by logging into the } \\
\text { Moodle account. Offline } \\
\text { email to user is available. }\end{array}$ \\
\hline Discussion Forum & $\begin{array}{l}\text { Discussion forums can be created in } \\
\text { a thread or blog. }\end{array}$ & $\begin{array}{l}\text { Instructors and students } \\
\text { are rated based on their } \\
\text { posts in the Forum. User } \\
\text { postings in the class Forum } \\
\text { can be emailed } \\
\text { automatically to the class } \\
\text { participants. Because } \\
\text { posting remains visible in } \\
\text { the Forum, those who do } \\
\text { not receive emails can } \\
\text { check for unread postings. } \\
\text { The user can create a } \\
\text { Forum for each class } \\
\text { workgroup and handle } \\
\text { each separately. }\end{array}$ \\
& &
\end{tabular}

The comparison of Moodle and Blackboard shows that the current e-learning systems use instant messages, emails and discussion forums as a knowledge transfer medium between knowledge seeker and knowledge provider. These communication systems just facilitate information transfer between knowledge seeker and knowledge provider. The communication modes used in Moodle and Blackboard have the provision to store the transferred knowledge but fail to quickly retrieve the required knowledge on demand by the knowledge seeker or knowledge provider. To solve this issue in e-learning environment, the researchers have developed a query management system for transferring knowledge between knowledge seeker and knowledge provider. The query management system has the capability to target experts and quickly retrieve the transferred knowledge on demand by the user or learner. The query routing mechanism used in the query management system contains clusters of expert groups on each topic or subject to which the query would be routed. The learners must select the appropriate expert group and route the query accordingly. The expert from the group must take 
charge of the assigned query and provide a solution to the query, and, in turn, the solution will be delivered to the user. This type of query routing mechanism fails if the user is new to the system and unaware of the query process, which may lead the user to receive an irrelevant or unworthy solution from the expert group because the expert who handled the query may be inexperienced or has less knowledge on the raised query topic, or the user may have routed the query to the wrong expert group due to human error.

To address these problems, we propose a dynamic query handling system that can receive the query from learners and automatically deliver it to the best subject expert available in the system. The user does not need to know the subject or expert group to which the query should be routed, and the experts in the dynamic query handling system are analyzed based on work performance and the solution delivery ratio that they uphold. The experts are ranked and re-ranked based on the performance that they maintain in the system, which retains the best performing expert as first priority on the topic or subject. The dynamic query handling system leads to a method for ranking experts, capturing expert knowledge, ensuring the best solutions for the query and providing a user-friendly environment to learners.

\section{Related Works}

Ahmad Kardan and Fatemeh Hendijanifard (2011) discussed the topic of finding subject experts for problem solving as an important issue in an e-learning environment. In an elearning environment, there is no direct approach to finding the superior individuals. The current methods of analyzing the discussions or considering the learner requires a large amount of data and contains limitations. In this work, concept maps are used to define the experts in an e-learning environment.

E-learning focuses on the use of computer and network technologies to create and deliver a rich learning environment that includes a broad array of instruction, information resources and solutions with the goal of enhancing individual and organizational performance. However, in this work, the term e-learning is used to encompass computer-based learning, computer-based training, technology-enhanced learning, technology-mediated learning, web-based education, or virtual learning environment. This topic has attracted considerable interest by providing a variety of benefits to learners, educational institutions, and organizations by removing the barriers of time and space in the development of knowledge and skill; providing just-intime learning, convenient access, and flexible learning processes; enabling real-time content updates while avoiding information overload; reducing travel, off-site training costs and time away from the job; and facilitating the interconnectivity of people for knowledge transfer (Ozdemir \&Abrevaya, 2007). 
Many schools have instituted course management software (e.g., Blackboard, WebCT, and Moodle) to complement traditional classroom-based instruction. Many empirical studies have been conducted to demonstrate how IT supports learning, improves student learning outcomes, enhances student information literacy, and increases the effectiveness of education management. Despite the variation in research findings, there is a consensus that substantial gains in student attainment are achievable if the use of IT in schools is planned, structured, and integrated effectively. However, to improve existing e-learning applications, smart learning environments must provide personal services to assist a learner in using, managing, and interacting with the learning system. A number of studies have investigated the use of intelligent tutoring techniques, that is, personalized learning interfaces and adaptive learning. These efforts generally emphasize technology development but exhibit little concern for effective instruction or pedagogy to enhance learning performance (Wang,Vogel, \& Ran, 2011).

Rajalakshmi and Wahidabanu (2011) proposed a model known as Info-Ca-Sh, a contributed knowledge portal of dynamic web content activities. The design flow serves as an exchange of knowledge among users by providing the users with a range of opensource tools. The tacit and explicit knowledge of the users are captured and externalized as a knowledge repository. The learning mechanism in higher education must be customized in accordance with the new requirements and current shifts in users, the culture and the economy. This effort requires new models for management of logical capital. Knowledge management can facilitate universities to find the appropriate people at the right time to make the best decisions using knowledge management systems. The knowledge management system refers to a system for customizing knowledge in organizations in terms of capture, storage information and broadcasting of knowledge. An approach based on elearning and its mechanism (Lloyd, 2003) is used to set up an organizational memory of the scientific, technical and administrative assets of the university and those interested in the construction of a warehouse of resources deliberately intended for training and for research.

The corporate sectors utilize the knowledge management techniques for sharing, capturing and storing of knowledge for effective usability. Koskinen (2003) discussed the introduction of a new model in which the business management can evaluate the type of role that tacit knowledge plays in their organizations. The model structure is discussed with four different systems, that is, memory, communication, motivational, and situational systems, and includes numerous factors that affect tacit knowledge utilization in organizations.

Senthil kumaran, Sankar, and Kiruthikaa (2014) discussed that the success of any elearning system depends on the quality and quantity of assistance provided to its students in the learning process. Hence, it is essential to analyze a student's academic skills to personalize the education that is provided both vertically and horizontally. 
The major component of the learning process is the result of the learner's collaborations and communications with subject experts. The collaborations in online group-based learning provide better opportunities to develop skills and knowledge compared with individual courses (Tahereh, Mahmood, Ahmad, \& Neda, 2011). The learning collaborations and communications with subject experts are highly practiced in corporate sectors by maintaining a knowledge portal for the stake holders.

A knowledge portal acts as an access tool for other information sources to provide internal and external information beyond the organization's own resources that can be made available to its staff. The portal also serves as a communication tool to enable individuals, teams and communities of practice to share and discuss ideas and knowledge (Venkata Subramanian, 2013). With the rapid development of globalization and technology, the importance of knowledge has also gradually increased. Whether enterprises can effectively create, accumulate, utilize and manage knowledge and convert knowledge into a competitive weapon has become a key to sustainable operation of modern enterprises (Chen, Lan, Lan, \&Hsu, 2014).

To combine the knowledge management concepts on e-learning environment, it is required that the e-learning system must contain a communication process integrated with knowledge clusters. To create a collaborative knowledge network, it is necessary to build a cluster of knowledge providers in the e-learning system. Fuzzy c-means algorithm is highly suggested to create clusters. In faster fuzzy clustering (also referred to as soft clustering), data elements can belong to more than one cluster, and a set of membership levels is associated with each element. These clusters indicate the strength of the association between that data element and a particular cluster. Fuzzy clustering is a process of assigning these membership levels and using them to assign data elements to one or more clusters. In many situations, fuzzy clustering is more natural than hard clustering. Objects on the boundaries between several classes are not forced to fully belong to one of the classes but rather are assigned membership degrees between 0 and 1 that indicate their partial membership. The fuzzy c-means algorithm (FCM) is used in computational geometry, data compression and vector quantization, pattern recognition and pattern classification. Fuzzy c-means (FCM) is an unsupervised clustering algorithm that has been applied to a wide range of problems involving feature analysis, clustering and classifier design. The FCM clustering, which constitutes the oldest component of software computing, is quite suitable for handling issues related to understanding patterns, incomplete/noisy data, mixed media information, and human interactions, and itcan provide approximate solutions (Yogeshwari \& Balamurugan, 2014). 


\section{Methodology}

The externalization of tacit knowledge in an e-learning environment takes place using the mechanism of capturing and sharing of knowledge with the right person at the right time. An e-learning system consists of learning contents in terms of documents, Power Point slides, videos, and so on. A well-defined elearning system provides communication between learners (knowledge seekers) and subject expertise (knowledge providers). The knowledge transferred between knowledge providers and knowledge seekers is considerably significant to maintain references for other learners. In addition, the significant queries raised by the knowledge seekers and directed to the knowledge providers will become key references for future learners.

Streamlining the process of knowledge transfer between knowledge seekers and knowledge providers requires a mechanism or knowledge management process for capturing, sharing and maintaining knowledge. E-learning is considered as an effective and well-utilized system that provides a well-defined and simple method of capturing the knowledge from experts and delivering to or sharing the captured knowledge with the relevant knowledge seekers. The query management systems used in an e-learning environment usually require categorized expert groups to facilitate the learners in posting queries to the relevant expertise group and receiving solutions. This process can be considered useful if the learner is highly familiar with the e-learning query management mechanism that routes their queries to the targeted expertise group.

The problem identified in this process is that the learner may not be able to find the targeted expertise group for the query if the learner is new to the e-learning query management system or unaware of the selective process. The learner may receive a nonvaluable solution for the raised query due to the incorrect routing of the query to the irrelevant expertise group or if the expert to whom the query has been assigned does not have updated knowledge on the particular topic. To address these issues, we propose a query management system known as the dynamic query handling system, which automatically takes the query from the learner to the targeted or relevant expertise and captures and shares the transferred knowledge among all learners.

In this paper, we propose an approach for externalization of expert tacit knowledge using a dynamic query handling system in an e-learning environment. In this approach, the knowledge seeker can raise a query to an expert; the user who raises the query does not require any type of prerequisite knowledge (i.e., the knowledge of to which expert group or individual expert the query must be routed or transferred). The knowledge seeker only needs to post the query, the query goes into an automatic processing flow mechanism that processes and routes the query to the most relevant expert in the system. The queries raised by users are also stored in the internal database and made searchable to other users. 
The diagram in Figure 1 shows the framework of the dynamic query handling system (DQHS) in an e-learning environment for automatic transfer of the query to the most relevant subject expertise rather than manual selection of an expertise group for routing the query. This mechanism uses two different paths of entry points into the system for users:

- knowledge provider or expert,

- knowledge seeker or learner.

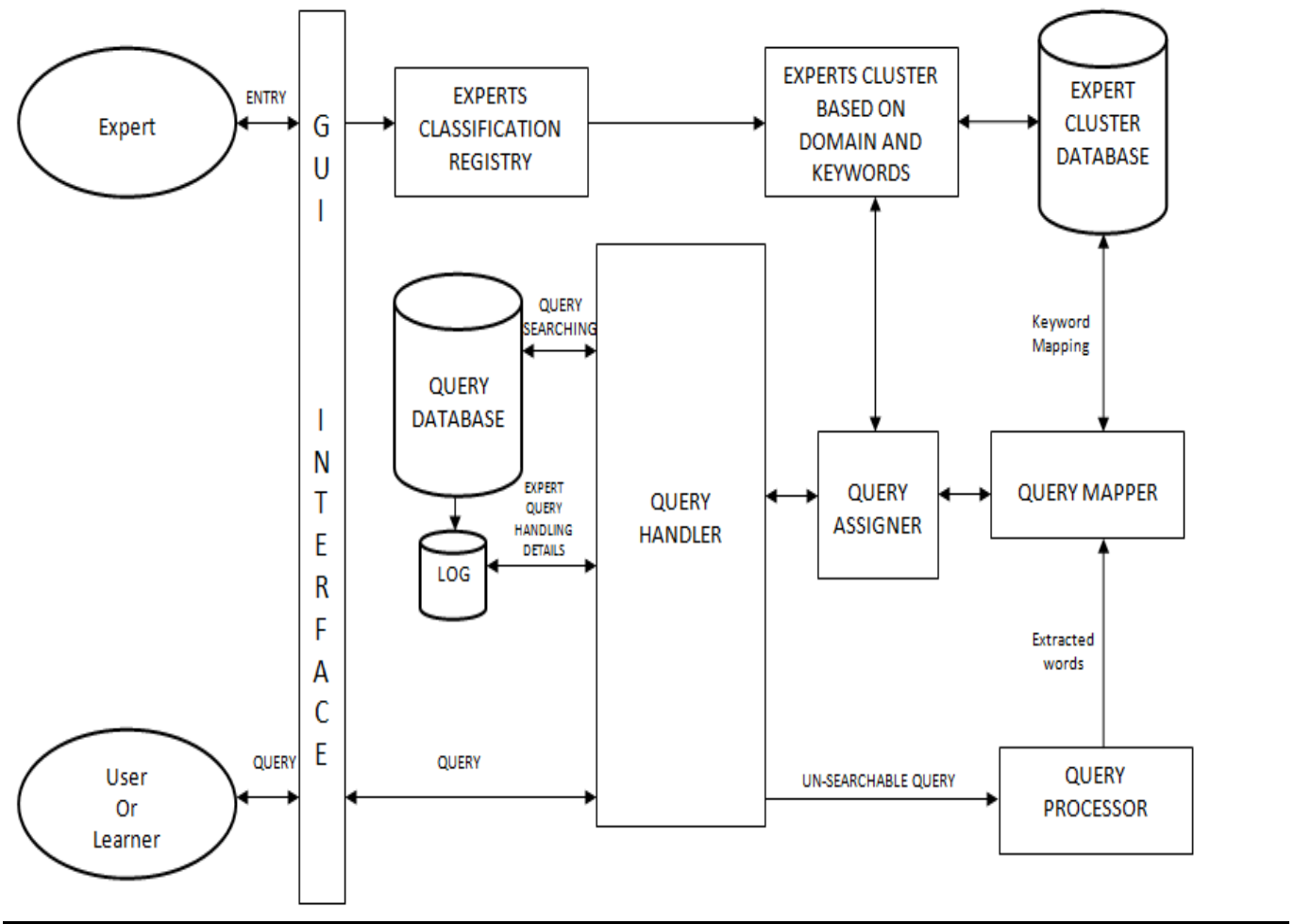

Figure 1. Dynamic query handling system.

The knowledge provider enters the system as a subject expert by providing specialized main keywords or main tags considered as the subject domain and sub-keywords or sub-tags considered as the specialized skill set in the appropriate domain. The subject experts are grouped by their main tags into three categories, that is, 1) expert, 2) moderately skilled expert, and 3) beginner, based on the skill level provided at the time of entry.

Each subject expert or knowledge provider is assigned a query bucket as a threshold for the maximum query limit. This query bucket is checked by the system before passing the query to the expert. If the query bucket reaches its threshold, the query will not be assigned to that particular expert, and instead, the system will look to the next level of 
expert in that particular domain for query assignment. The query is first routed to the expert level of subject expertise for query resolution. If all expert-level subject experts are engaged with existing queries and have reached their maximum levels of query assignment, then the query will be auto-redirected to the moderate level of subject experts and subsequently to the beginner level of subject experts in accordance with the query bucket thresholds. The dynamic query handling system consists of the following components for query processes:

- expert classification registry,

- expert cluster based on domain keywords,

- query handler,

- query processor,

- query mapper,

- $\quad$ query assigner.

\section{Expert Classification Registry (ECR)}

The expert enters the dynamic query handling system using the ECR process. The ECR process asks the expert to provide their specialized main keywords or main tags (considered as the subject domain) and sub-keywords or sub-tags (considered as the specialized skill set in the appropriate domain). The ECR process also requires the expert to enter a skill level in the domain, that is, expert, moderately skilled expert, beginner. These expertise levels are used by the dynamic query handling system for query transfer to the best expert in the domain. The ECR process also requires the experts to enter their expertise levels for their sub-keywords or sub-tags. This information is also used to map the most knowledgeable expert in the domain for userraised queries. This process also asks the expert to provide a query threshold limit for query assignment.

\section{Expert Cluster Based on Domain Keywords (ECDK)}

The ECDK mechanism uses the outcomes of ECR to create clusters of experts based on their domains and sub-domains. If the domain cluster already exists, the expert is added into the existing clusters, and the sub-keywords or sub-tags are merged into the expert specialization list.

\section{Query Handler}

The query handler is the main component and is interlinked with other components in the dynamic query handling system. The raised query from the learner or user enters the query handler, and the query handler checks the query within the query database 
and if a pre-existing query with solution is not available in the database, it transfers the query to the query processor component. Thus, the query handler operates as a search mechanism and gives the results to the user or learner.

\section{Query Processor}

The query processor component delivers the raised query to the natural language processing parser, where the query is filtered by removing the stop-words. As a result, the extracted words are obtained. These extracted words are transferred to the query mapper.

\section{Query Mapper}

The extracted words are entered into the process of word mapping using the query mapper and the fuzzy c-mean genetic algorithm process checks the higher feasibility of mapping the words with expertise keywords. Once a higher match is found, the query will be mapped to the concerned expertise group to route or transfer the query to the particular expert.

\section{Query Assigner}

Once a subject expertise group is mapped, the query assigner checks the meta-data generated from the query database and validates it based on the number of queries handled by the expert and the number of users who accepted the query solution provided by the expert. Next, the processed validation data are used by the ranking algorithm to re-rank the experts based on the performance in the system. The query assigner uses the result of the re-ranked expert list and finds the high performing expert for the particular query, and the user query is transferred to that particular expert. On the other hand, the query assigner component also checks the query bucket threshold limit before assigning the query to the target expert, and if the particular expert query bucket has reached the threshold limit, the query will go to the next best expert in that particular domain. The dynamic query handling system frequently assesses the performance of the experts using the number of queries handled by the expert, the number of solutions accepted by the user and the user ratings for the expert. These constraints are applied to auto re-rank the experts in their subject domains.

\section{Implementation}

The implementation of the dynamic query handling system (Figure 1) is distributed into three combinations of processes:

- expert enrolment,

- expert segregation, 
- user query mapping.

Expert enrolment is a feed process used by the dynamic query handling system to collect the information from the expert and store it in the database.

\begin{tabular}{|c|c|c|c|c|c|c|c|c|c|c|}
\hline \multirow{2}{*}{ 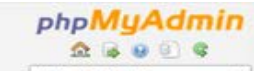 } & \multicolumn{10}{|c|}{ D querymodei = Estatiregster } \\
\hline & Drowse & $\Rightarrow s$ & Structure & 45 & SQL & Search \$i & Insert $\rightarrow$ Exp & kport $\Leftrightarrow$ & Import & Ope \\
\hline \multirow{9}{*}{ 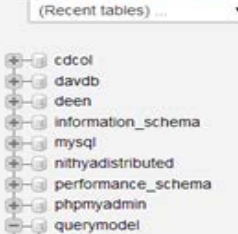 } & a $2 \mathrm{Ean}$ ? & $\$$ i copy & $\theta$ Delete & 11 & 1011 & JavaExpert11 & jexpert11 & 123 & & $1 \mathrm{lec}$ \\
\hline & (1) $\delta$ Edt $\bar{x}$ & \$i copy & $\Theta$ Delete & 12 & 1012 & JavaExpert12 & jexpert12 & 123 & & $1 \mathrm{lec}$ \\
\hline & (-) Eant & \$i copy & D Delete & 13 & 1013 & JavaExpert13 & jexpert13 & 123 & & $1 \mathrm{lec}$ \\
\hline & (1) Edr F & ॠi copy & (9) Delete & 14 & 1014 & JavaExpert14 & lexpert14 & 123 & & 2 sec \\
\hline & - Edat & \$i Copy & $\theta$ Delete & 15 & 1015 & JavaExpert15 & jexpent15 & 123 & & 2 lec \\
\hline & D. Edit & \$i copy & $\theta$ Delete & 16 & 2001 & Os_Startert & OS_starter01 & 123 & & 2 lec \\
\hline & - $\&$ Edt F & $\$ i$ Copy & D Delete & 17 & 2002 & Os_Starter2 & os_starter02 & 123 & & $1 \mathrm{lec}$ \\
\hline & $\nabla$ Edit $\bar{x}$ & Fic copy & $\theta$ Delete & 18 & 2003 & Os_Stanter3 & OS_stanter03 & 123 & & $1 \mathrm{lec}$ \\
\hline & (-) Ean F & \$i Copy & O Delete & 19 & 2004 & Os_Starter4 & Os_startero4 & 123 & & $2 \mathrm{sec}$ \\
\hline \multirow{8}{*}{ 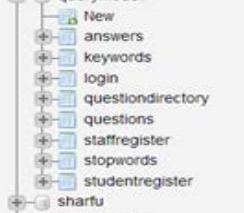 } & (1) Edi & $\$ \approx$ copy & $\theta$ Delete & 20 & 2005 & Os_starter5 & OS_starter05 & 123 & & 1 lec \\
\hline & ㄷ. Eat $\$$ & \$i Copy & O Delete & 21 & 2006 & Os_Moderator6 & os_moderator06 & 123 & & $1 \mathrm{lec}$ \\
\hline & 口. OEdt : & \$i Copy & $\Theta$ Delete & 22 & 2007 & Os_Moderator7 & OS_moderatoro7 & 123 & & $2 \mathrm{lec}$ \\
\hline & • $D$ Ean & $\$ i$ copy & A Delete & 23 & 2008 & Os_Moderator8 & os_moderatorcs & 123 & & 1 lec \\
\hline & C. Eoli * & ॠi copy & $\theta$ Delete & 24 & 2009 & Os_Moderatorg & Os_moderatoros & 123 & & 1 vec \\
\hline & - Edin * & Fi Copy & $\theta$ Deiete & 25 & 2010 & Os_Moderatorto & OS_moderator10 & 123 & & 1 lec \\
\hline & Q. $O$ Eat 5 & $\$ i$ copy & Q Delete & 26 & 2011 & Os_Expert1 & os_experto1 & 123 & & 1 lec \\
\hline & $\square$ Ean & \$i copy & O Delete & 27 & 2012 & Os_Expert2 & os_expert02 & 123 & & $2 \mathrm{lec}$ \\
\hline \multirow{5}{*}{$\begin{array}{l}+-3 \text { students_db } \\
+-3 \text { test } \\
+-3 \text { webauth }\end{array}$} & D Edit $\bar{x}$ & \$i copy & $\theta$ Delete & 28 & 2013 & Os_Expert3 & Os_Expert03 & 123 & & 2 lec \\
\hline & ㅁ Edin ₹ & \$i Copy & - Delete & 29 & 2014 & Os_Expert4 & Os_Expento4 & 123 & & $1 \mathrm{hec}$ \\
\hline & (1) 2 Edit & \$్je copy & $\theta$ Delete & 30 & 2015 & Os_Expents & Os_Expent05 & 123 & & $1 \mathrm{lec}$ \\
\hline & ㅁ. Eot : & \$i copy & (9) Delete & 31 & 3001 & Sq!_Starter1 & Sql_Stanter01 & 123 & & $1 \mathrm{sec}$ \\
\hline &  & \$i copy & $\Theta$ Delete & 32 & 3002 & SqI_Stanter2 & Sql_starter02 & 123 & & $1 \mathrm{lec}$ \\
\hline
\end{tabular}

Figure 2. Screenshot of expert registry.

The staff registry (Figure 2) displays the expert enrolled information stored in the internal database. This registry contains unique information, including the expert user ID, expertise level (i.e., expert in topic, moderate in topic, and beginner in topic), expert email ID, and so on. These enrolled data are used at the time of query transfer confirmation.

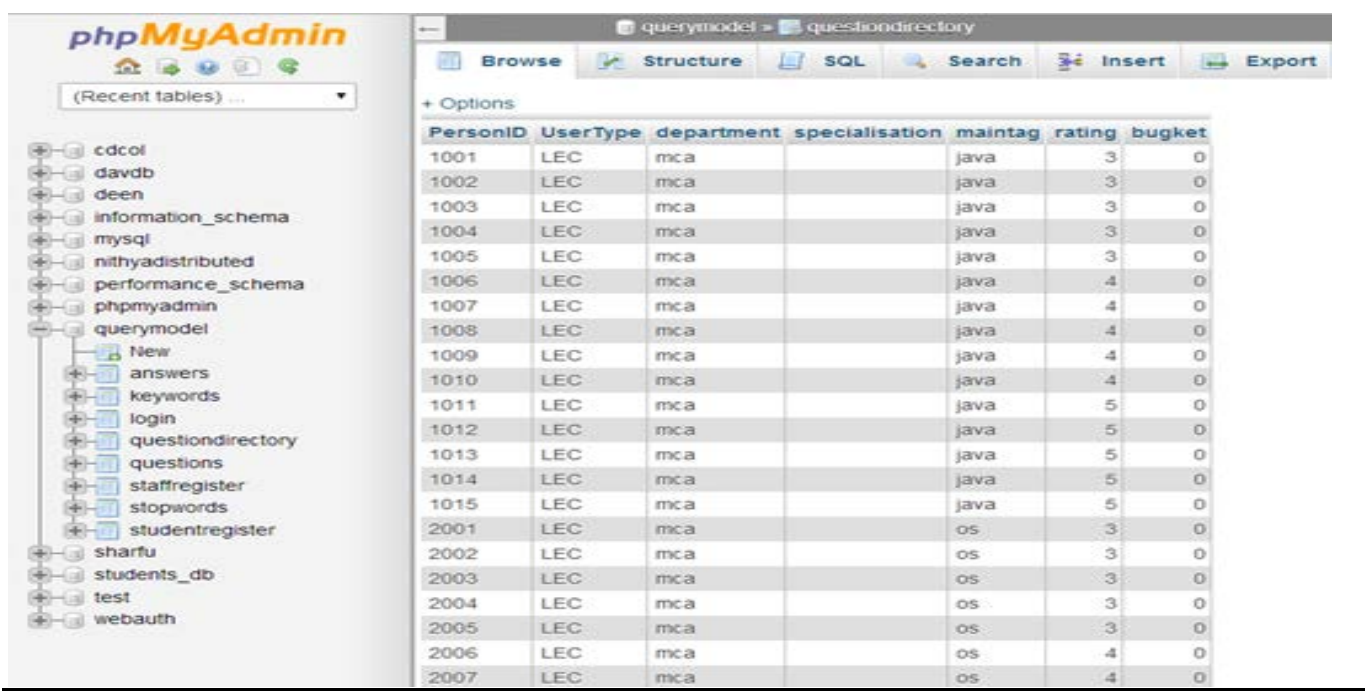

Figure 3. Expert domain directory screenshot. 
The expert segregation process uses the expert domain directory (Figure 3) contains the expert ids, expert domains or main-tags, expert ratings and expert bucket threshold limits. The expert id field in the database is used to determine the unique identity of the expert, the expert domains or main-tags field is used to choose the expert specialization domain or subject area, and the expert rating field is the value for expert level of specialization (expert on subject topic, moderate knowledge of subject topic and beginner in subject topic). These field values are used by the dynamic query handling system to make a decision for transfer of a query to the expert. The system also checks the expert rating field to pick the best expert for the query assignment. The expert bucket field value is checked by the system before query assignment to an expert. If the query bucket of an expert is full or reaches the threshold limit, then the system will check for the next best expert for the query assignment.

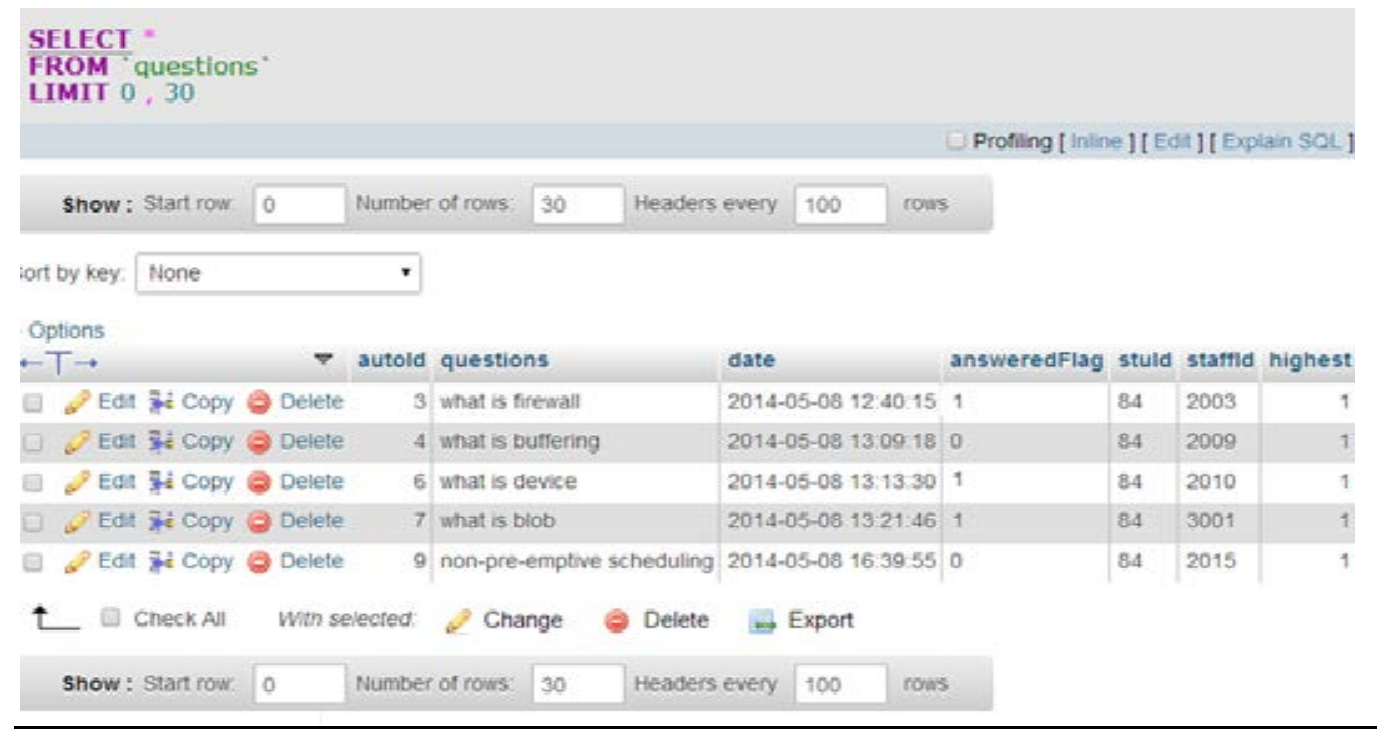

Figure 4. Question directory screenshot.

The question directory screen shot (Figure 4) shows the user query mapping done by the system. The answer flag field shows the number of answers given by the experts and also the staff ID of the person to whom the questions are mapped and assigned.

The mathematical measurements derived for the query transformation are given below.

$$
\begin{aligned}
& \mathrm{Qt}=\left\{\begin{array}{l}
((\mathrm{ky} \cap \mathrm{ed}) \text { and }(\mathrm{sw} \cap \mathrm{sd})) \rightarrow \mathrm{xp}((\mathrm{er}>\mathrm{oxp}) \text { and }(\mathrm{bc}<\mathrm{th})) \\
\mathrm{Qt}=\text { Query Transformation }
\end{array}\right. \\
& \mathrm{Ky}=\text { Keywords } \\
& \text { ed= Expert Domain }
\end{aligned}
$$




$$
\begin{aligned}
& \mathrm{sw}=\text { Sub-keywords } \\
& \mathrm{xp}=\text { Expert } \\
& \mathrm{er}=\text { Expert Rating } \\
& \mathrm{oxp}=\text { Other Expert in the same domain } \\
& \mathrm{bc}=\text { Query bucket } \\
& \text { th= Query bucket threshold }
\end{aligned}
$$

The query (Qt) is transferred to the expert (xp), and the keywords (ky) extracted from the query using the NLP parser are used to map or match with the expert domain list (ed), that is, the intersection of the keywords and expert domain list (ed) contains a common word, and the sub-keywords (sw) for the query also are mapped on the subdomain list (sd). The resultant expert's rating (er) must be greater than those of other experts (oxp), and the query bucket (bc) must be less than the threshold limits (th).



Your Query Mapping

Figure 5. Query posting screen shot.

The user query posting screen shot (Figure 5) shows that the system presents a userfriendly GUI for ease of use so that posters can send the query to the expert without checking expert lists or expert groups to which the query is more suitable for transfer.

The algorithm generated for the query transformation is described below:

Step 1: User raises query.

Step 2: Query is processed using the NLP parser (Lucene). This process removes stopwords and yields informative words or extracted words. 
Step 3: The extracted words (EW) are compared with expert stored main-tags (MT) and sub-tags (ST), i.e., $\mathrm{EW}=\mathrm{MT}$ or ST

Step 4: Once a match is found between the extracted words and the expert keywords, the user query is mapped with the expert and expert domain.

Step 5: Once a query is mapped with expert $i$,

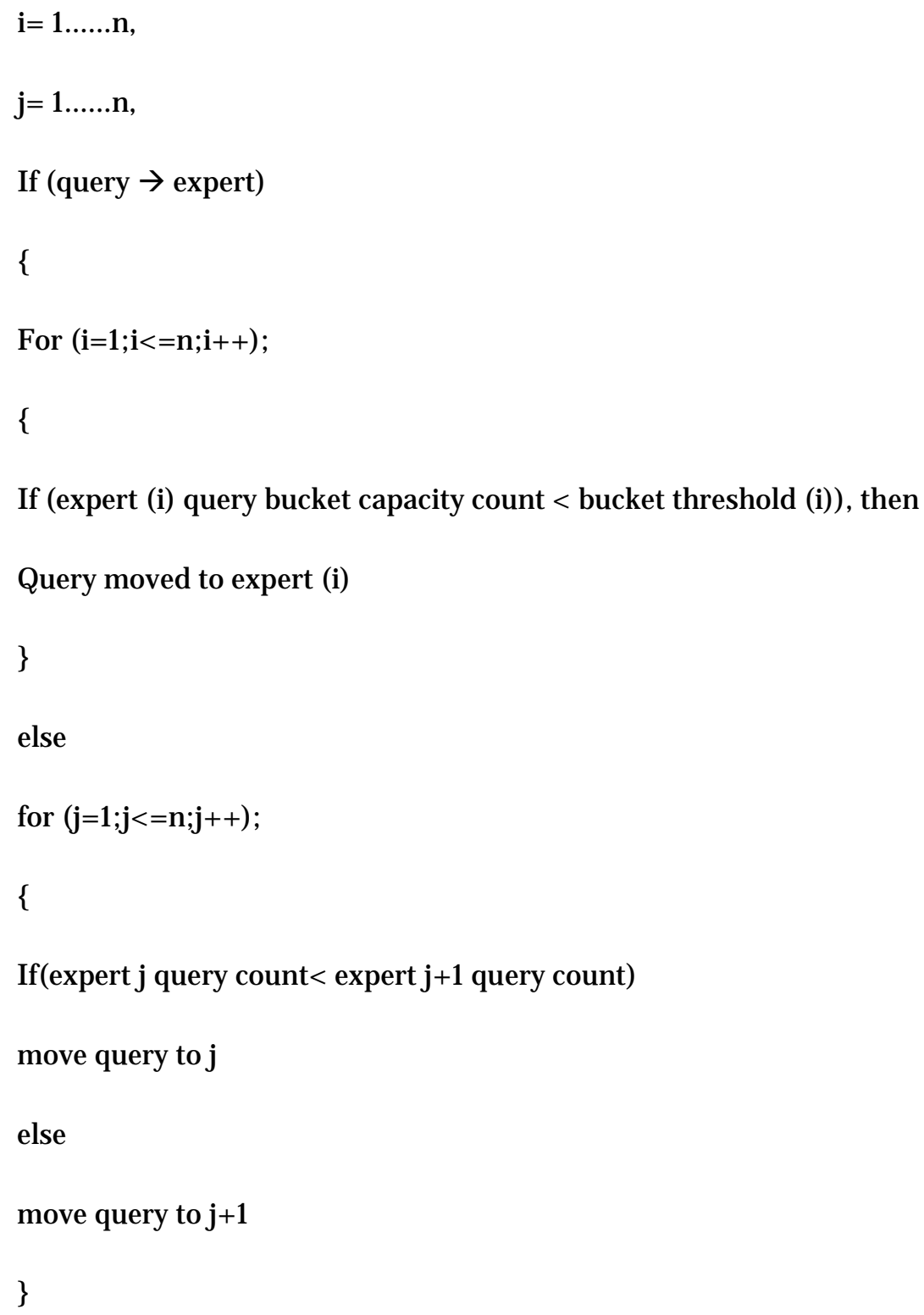

Step 6: If (query<> experts (keywords), then Move query to dispatcher. 
The algorithm generated for query transformation indicates that user queries are passed through the NLP parser to remove stop words and obtain extracted words, which in turn are compared with the expert stored keywords for the mapping processes, and the mapped expert's query bucket threshold limit is checked before transmitting the query to the concerned expert. If the expert's query bucket is full or the threshold has been reached, then the query will be redirected to the next expert. If the query is not matched with any of the expert keywords, then the query will be moved to the dispatcher. The dispatcher or the administrator will divert the query to an expert by manually finding the expert or the query will be forwarded to the appropriate knowledge management team to address the issue and resolve it.

A functionality audit of the system was carried out with 100 users using three subjects, namely, operating system, J ava and networks.

Table 2

Total Queries With Query Relevance

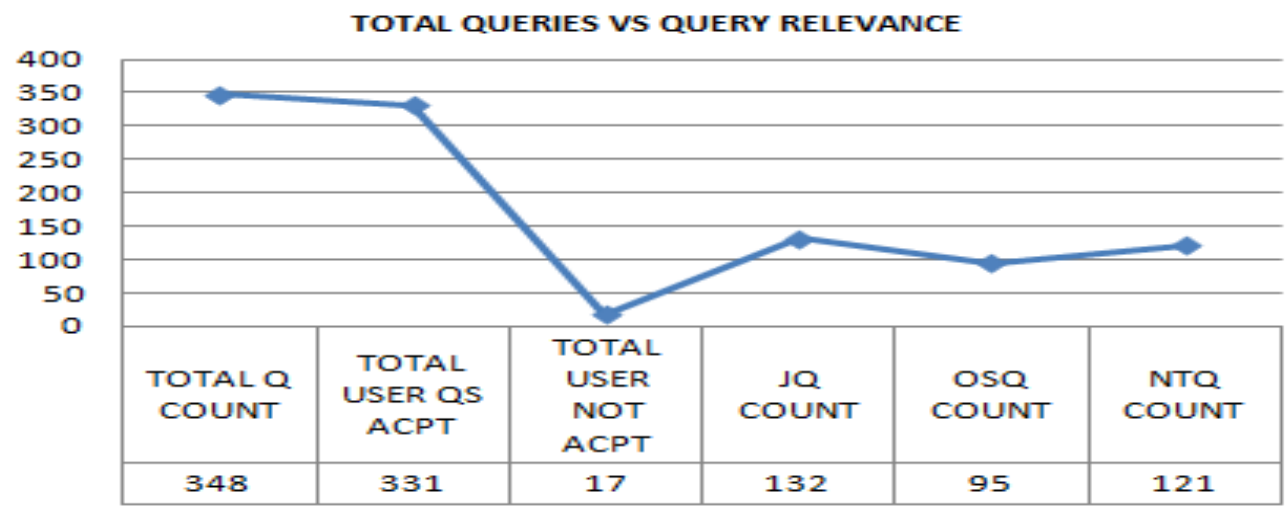

Q $\rightarrow$ Query, QS $\rightarrow$ Query Solution, ACPT $\rightarrow$ Accepted, J Q $\rightarrow$ J ava Query, OSQ $\rightarrow$ Operating System Query, NTQ $\rightarrow$ Network Query.

The results shown in the above graph indicate that the total number of queries raised by 100 users is 348 for which 331 solutions were provided by the experts and accepted by the users as worthy, and 17 solutions were rejected or unaccepted by users. The total Java-related queries raised by users was 132, the total number of queries for the operating system was 95 and that of networks was 121 . The result shows that all queries are assigned properly to the experts out of which 17 queries are marked unworthy. 


\section{Conclusion and Future Direction}

The externalization of tacit knowledge in an e-learning environment takes place using the mechanism of capturing and sharing of knowledge with the right person at the right time. The framework of a dynamic query handling system has the significance of taking a query from a user to an appropriate expert automatically, without the use of manual routing parameters such as subject expert IDs or expert group IDs, and so on. The results reveal that the queries raised by users are transferred to the appropriate experts automatically using the main-tag and sub-tag entries provided by the expert. The system also checks the expert query bucket threshold before placing a query in the expert queue to maintain the correct expert workload. These mechanisms offer a path to reaching a potential expert for solution of a problem and the opportunity to collect valuable knowledge from the expert and store it in the knowledge repository. The stored knowledge in the knowledge repository is searchable by users to find relevant answers for the issue or problem before querying an expert. We claim that this approach can satisfy most of the needs of knowledge seekers by directly connecting to domain expertise for collection of tacit knowledge and transforming it into externalized knowledge. The proposed query management system can be limited to query mapping with experts based on keyword matching or can be improvised to handle queries by extracting the meaning of the query and subsequently mapping it to the appropriate expert based on the query meaning. Future work could include connecting the dynamic query handling system with search engines and adding agent-based search services to use the knowledgeable experts available on the internet for query resolution or knowledge transfer. Search engine connectivity will extend this query management system into the cloud computing environment, which will pose challenges related to higher storage, $24 \mathrm{X} 7$ system up-time, large numbers of open sessions, archive backups, and compatibility with various open source software and licensed software. 


\section{References}

Kardan, \& Hendijanifard.(2011). Expert finding system in e-learning: Using concept maps. International J ournal of Information \& Communication Technology, 3(1), 73-81.

Manongga, D., Utomo, W.H., \& Hendry.(2014). E-learning development as public infrastructure of cloud computing. J ournal of Theoretical and Applied Information Technology, 62(1), 54-59.

Venkata Subramanian, D. (2013). Relationships between factors for evaluating knowledge sharing portals. International J ournal of Engineering and Technology, 5(3), 2248-2256.

Koskinen, K. U.(2003). Evaluation of tacit knowledge utilization in work units. J ournal of Knowledge Management, 7(5),67-81.

Lloyd, J . W. (2003). Logic for learning: Learning comprehensible theories from structured data. Springer-Verlag.

Mirsaeedghazi, T., Mahmood, K., Ahmad, K., \&Neda, E. (2011). A framework for learner preparation and support in academic e-learning environment. International J ournal of Information \& Communication Technology, 3(1),3341.

Chen, P-C. Lan, P-S., Lan, H., \&Hsu, H-Y.(2014). Dynamic effect of knowledge management system on school management. J ournal of Theoretical and Applied Information Technology , 61(2),249-253.

Ozdemir, O., \&Abrevaya, J . (2007). Adoption of technology-mediated distance education: A longitudinal analysis. Information \& Management, 44(5), 467479.

Rajalakshmi, S., \& Wahidabanu, R. S. D.(2011). Sharing and capturing tacit knowledge in higher education- The Info-Ca-SH. International J ournal of Computer Theory and Engineering, 3(3),365-368.

Senthil kumaran, V., Sankar, A. \& Kiruthikaa, K. (2014). Community based recommendation in e-learning systems. J ournal of e-Learning and Knowledge Society, 10(1), 51-61.

Wang, M., Vogel, D., \& Ran, W.(2011), Creating a performance-oriented e-learning environment: A design science approach. Information And Management, 7, 260- 269. 
Yogeshwari, T., \& Balamurugan, S. (2014). A novel approach for user search results using feedback sessions. International J ournal Of Modern Engineering Research, 4(5), 70-75.

(c) Khan and Khader

\section{Athabasca University $\mathbf{I}$}

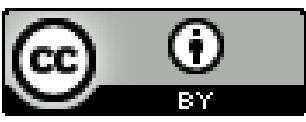

This work is licensed under a Creative Commons Attribution 4.0 International License. 